\section{MS}

médecine/sciences $1996 ; 12: 1331-3$

\title{
PEUT-ON INTÉGRER L'EMBRYON HUMAIN DANS LE CHAMP MÉDICAL ET SCIENTIFIQUE?
}

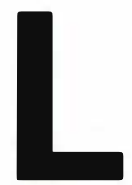

a maîtrise in vitro de la fécondation et du développement embryonnaire jusqu'au stade blastocyste conduit obligatoirement à se préoccuper de la qualité des embryons ainsi conçus. Par ailleurs, prélever une ou deux cellules très précocement sur l'embryon ne perturbe pas les capacités de développement, et il existe des techniques de plus en plus précises et fiables pour identifier des altérations chromosomiques ou géniques à ce stade; il était donc prévisible que cette approche soit envisagée par et pour des couples chez lesquels il existe un risque important et connu de transmission d'une maladie héréditaire identifiable.

Les premières naissances d'enfants conçus in vitro et pour lesquels un diagnostic génétique avait été fait avant l'implantation ont eu lieu au Hammersmith Hospital à Londres en 1990 [1]. Des séquences spécifiques du chromosome Y avaient été détectées sur les embryons conçus in vitro pour cinq couples chez lesquels existait un risque de transmission de maladie liée à l'X (adrénoleucodystrophie et retard mental). Depuis, la même démarche a été répétée pour détecter des maladies dues à des mutations spécifiques comme la mucoviscidose, la myopathie de Duchenne, la maladie de Tay Sachs ou des maladies liées au sexe en utili- sant l'hybridation in situ fluorescente ou la PCR (voir l'article de Viville $e t$ al. p. 1378 de ce numéro [2]).

La très bonne synthèse faite par ces auteurs montre que ce type de diagnostic est encore peu répandu et n'a par ailleurs jamais été utilisé pour des raisons de convenance. Elle analyse aussi la complexité des méthodes, les difficultés et les risques d'erreur. Il est évident, cependant, que la multiplication des sondes disponibles, les progrès des méthodes de préamplification, le recours à la micro-injection des spermatozoïdes dans l'ovocyte et à l'amplification des séquences microsatellites vont offrir des techniques de plus en plus sûres pour des diagnostics de plus en plus nombreux.

Aucune équipe française ne figure parmi les quatorze dont le bilan est rapporté dans l'article de Viville et al. C'est d'autant plus étonnant que la France est un des très rares pays à avoir autorisé ce type de diagnostic par voie législative [3]. L'explication est simple, aucun des décrets d'application nécessaires à l'organisation de cette activité n'a été publié. Cette absence, qui est la même pour les études sur l'embryon, reflète incontestablement le malaise sinon les réticences des autorités civiles de notre pays chaque fois que l'embryon humain est en cause.

Réunissant trois actes : une procréation médicalement assistée, une intervention sur l'embryon et un diag- 
nostic génétique, qui suscitent chacun de très nombreuses questions éthiques, il est compréhensible que le diagnostic génétique préimplantatoire (DPI) soit l'objet d'un débat très approfondi. Malheureusement le débat s'appuie plus souvent sur des convictions et des affirmations à l'emporte-pièce que sur des faits. Il prend quelquefois des allures très polémiques comme dans la libre opinion de Testart et Sèle publiée dans ce numéro [4].

Ces auteurs me semblent avoir raison sur deux points. Le questionnement est beaucoup plus éthique que technique et assimiler le DPI à un diagnostic prénatal ultraprécoce est sans doute une erreur. Si la finalité est la même : éviter pour des parents, d'avoir un enfant gravement malade ou handicapé qu'ils ne se sentent pas capables de mettre au monde, les conditions de réalisation et les enjeux tant du point de vue des couples que des médecins sont extrêmement différents.

Les conditions prévues dans la loi pour la réalisation du DPI sont très précises et très strictes. Cet acte "exceptionnel... ne peut être effectué que lorsqu'a été préalablement et précisément identifiée, chez l'un des parents, l'anomalie ou les anomalies responsables d'une... maladie génétique d'une particulière gravité reconnue comme incurable... que le couple a une forte probabilité de transmettre à l'enfant". Testart et Sèle jugent ces dispositions remarquables et ne s'opposeraient pas au DPI si elles étaient appliquées dans la transparence. Pourquoi ne le seraient-elles pas? La loi devant faire l'objet d'un nouvel examen en 1999, il aurait été utile que les parlementaires puissent disposer d'autres critères d'appréciation que ceux donnés par l'expérience d'équipes travaillant dans d'autres pays et ayant des critères différents.

Pourtant Testart et Sèle expriment, non sans vivacité, une opposition très forte au DPI. Ils craignent que la banalisation de la procédure conduise les praticiens à répondre sans limite au désir d'enfant idéal. Pour eux, accepter le principe du tri des embryons ne serait que le point de dérive inéluctable conduirait à instrumentaliser l'homme en sélectionnant le meilleur embryon. Le DPI ne serait que l'application à des individus d'un consensus d'exclusion sur des critères biologiques caractérisant l'eugénisme. L'argumentation utilisée pour démontrer cette théorie s'appuie sur des formules fortes et souvent chocs : "cette technique est la seule susceptible d'un réel effet eugénique ", " elle met en cause le principe d'altérité et la définition de la personne humaine ", " nous y voyons la remise en cause de concepts fondamentaux qui fondent l'humanisme ", " la dérive est inscrite dans la demande ", etc.

Les risques de dérive du DPI ne peuvent être niés, mais sont-ils si importants ? Sont-ils spécifiques du DPI ? Ne peuvent-ils vraiment être maîtrisés ?

Le tri des embryons est une pratique quotidienne et obligatoire des laboratoires de fécondation in vitro (FIV). Du $1^{\text {er }}$ janvier au 31 juillet 1996, 2868 embryons ont été conçus par FIV au laboratoire de l'hôpital Cochin. Parmi ceux-ci, 131 (4,6\%) contenaient plus de 2 pronucleus, 16 à 18 heures après l'insémination ou l'injection des spermatozoïdes. Ces embryons polyploïdes sont capables de se développer in vitro ou in vivo, au moins pendant un certain temps, mais il ne viendrait à l'idée de personne de les transférer dans l'utérus de la femme. Les biologistes ont donc procédé à un tri embryonnaire fait sur critères génétiques (la ploïdie), et le développement de ces embryons a été interrompu. Les 2737 embryons diploïdes " sélectionnés " ont été remis en culture. Le lendemain, ils ont été classés selon des critères morphologiques tenant compte du nombre et de l'aspect des blastomères, et de la quantité de fragments cellulaires. La relation entre la "qualité embryonnaire " et les chances de développement in vivo étant bien démontrée, il a été procédé à ce stade à un deuxième tri embryonnaire conduisant à sélectionner les embryons ayant le meilleur aspect afin de les transférer dans l'utérus de la femme et de les congeler quand le couple a donné son consentement. Le développement des autres a été interrompu. Ce fut le cas pour 499 embryons $(17,4 \%)$ pendant la période considérée à Cochin. Ce tri embryonnaire sur critères génétiques et morphologiques est pratiqué dans tous les laboratoires de FIV quels qu'ils soient, y compris dans les hôpitaux catholiques ou les pays qui refusent la congélation embryonnaire. Il ne peut en être autrement.

Bien sûr, on pourrait objecter que ce tri n'a rien à voir avec le DPI car il ne s'agit ici que de sélectionner les embryons ayant les meilleures chances de se développer en un enfant vivant et normal du point de vue de sa ploïdie, alors que le diagnostic génétique chercherait à éliminer un "enfant potentiel " porteur d'une tare génétique mais viable ou encore à sélectionner des embryons en fonction de tel ou tel trait jugé attrayant ou le meilleur.

Le risque de dérive est possible mais le DPI est loin d'être le seul ou le meilleur moyen de réaliser un désir d'enfant choisi en vue de satisfaire des pulsions eugénistes. Dans certains pays, le diagnostic prénatal suivi d'un avortement est malheureusement largement utilisé pour choisir le sexe de l'enfant à naître au point de modifier de manière importante le sex ratio à la naissance avec toutes les conséquences sociales et démographiques que cela implique [5]. Surtout, le diagnostic et le tri préconceptionnel des gamètes sera sans doute prochainement beaucoup plus efficace. Le contenu chromosomique de l'ovocyte non fécondé peut être indirectement déterminé par l'analyse du globule polaire [6].

Récemment, Levinson et al. ont séparé des spermatozoïdes humains porteurs du chromosome X ou du chromosome Y par cytométrie en flux sur la base des $2,8 \%$ de différence du contenu en ADN [7]. Le nombre de spermatozoïdes sélectionnés était très faible et il a fallu recourir à une FIV pour concevoir l'enfant. Nul doute qu'avec quelques améliorations techniques, il sera bientôt possible de sélectionner suffisamment de spermatozoïdes pour concevoir aisément un enfant du sexe choisi par simple insémination artificielle.

Parallèlement, les méthodes de typage génique du spermatozoïde pro- 
gressent rapidement [8]. Les modifications qu'elles induisent au niveau des spermatozoïdes rendent actuellement impossible leur utilisation dans un but thérapeutique mais, une fois résolu le problème de l'accès à l'ADN sans modification de la structure et des fonctions cellulaires et nucléaires, il en sera tout autrement. Les moyens de diagnostic devenant de plus en plus accessibles, va-t-on assister à une demande illimitée de conception d'enfants pour satisfaire les fantasmes des parents? Rien ne permet de l'affirmer. L'expérience des centres qui proposent de choisir le sexe des enfants est intéressante à analyser. Dans une clinique de fertilité de la Nouvelle-Orléans qui propose la sélection du sexe dans un programme d'insémination, la méthode est demandée par seulement $5 \%$ des couples [9]. Dans une "Gender Clinic " qui s'est ouverte en 1993 à Londres, $37 \%$ des 809 couples ayant consulté avaient été traités après 2 ans. Si la presque totalité des couples d'origine indienne souhaitait un garçon, la majorité des couples d'origine européenne souhaitait une fille. Surtout, ils avaient tous un nombre élevé d'enfants du sexe non recherché et la demande apparaissait surtout comme le moyen de compléter une famille avec un enfant supplémentaire du sexe différent de celui des enfants déjà existants [10]. Bien que marginales, ces demandes peuvent néanmoins être considérées comme inacceptables, mais doit-on pour autant les assimiler à l'eugénisme ? Rien n'est plus complexe que le désir d'enfant. Rares sont les enfants qui n'existent pas dans l'imagination de chacun bien avant qu'ils ne soient nés. Comme le dit Tomkiewicz, "le fantasme (de l'enfant rêvé, de l'enfant parfait) n'aura pas de répercussion trop dramatique sur notre avenir : il coexistera toujours avec le courant humaniste et d'amour qui incite les familles à accepter l'enfant tel qu'il est, voire à en adopter de plus faibles... Je crains par contre un avenir très sombre si le fantasme de l'enfant parfait s'empare des chercheurs, des praticiens et des pouvoirs politiques "[11]. Le risque principal serait donc du côté des professionnels et de la société.
Affirmer que les dérives sont inéluctables si le principe même du DPI est accepté apparaît non seulement inopérant mais contraire à toute tradition en matière de recherche et de pratique médicale. Comment pourrait-on s'engager dans quelque activité que ce soit si des conséquences potentiellement dangereuses ou indésirables devraient conduire à en refuser l'existence même? La mauvaise utilisation des techniques et des thérapeutiques ou le comportement inapproprié de professionnels peuvent se rencontrer dans tous les domaines de la médecine, de la chirurgie cardiaque à la prescription des tranquillisants en passant par la chirurgie esthétique et bien d'autres traitements. Doit-on pour autant en refuser le principe ?

L'hypothèse de la pente glissante est discutable. On pourrait lui substituer celle de l'escalier, la décision de passer d'une marche à l'autre pouvant être prise en s'appuyant sur la réalité des possibles et de l'expérience. Rien n'empêche d'ailleurs de dresser des barrières infranchissables entre les marches quand c'est nécessaire. C'est ce qu'a fait la loi française.

Les possibilités offertes par l'assistance médicale à la procréation, par le choix des gamètes et des embryons qu'elle autorise bouleversent certainement les repères sociaux et moraux en matière de procréation [12]. Des choix qui étaient impossibles ou appartenaient à l'intimité du couple sont maintenant partagés avec l'institution médicale. De nouveaux choix apparaissent possibles.

La génétique de la procréation conduit quelquefois à éviter la transmission de maladies héréditaires, d'autres fois elle favorise la diffusion de certaines mutations comme celles du gène CFTR quand on aide un homme ayant une agénésie bilatérale des canaux déférents à devenir père par micro-injection de ses spermatozoïdes dans les ovocytes de sa femme [13].

Les couples sont souvent confrontés à des choix difficiles pour concevoir leurs enfants, surtout quand cela entraîne médicalisation de la procréation, don de gamète, diagnostic génétique, interruption de grossesse. Ils doivent pour cela recevoir une information aussi précise que pos- sible sur les possibilités mais aussi sur les avantages, les inconvénients et les enjeux des différentes méthodes. Ce conseil génétique ne peut être une "orientation " génétique ou la promotion de normes ou de convictions, il ne doit rester qu'une aide à la décision. La responsabilité que doit exercer le médecin, d'ordre déontologique, appartient au domaine de l'éthique [14]. Elle ne peut être totalement définie par des réglementations, ni par des programmes informatiques si parfaits et sophistiqués soient-ils. Elle peut s'appuyer sur la confrontation de points de vue variés et multidisciplinaires. Elle peut être évolutive comme le montre l'expérience de la commission de génétique de la fédération des CECOS qui traite des sujets du même ordre dans le domaine du don de gamètes.

Il serait peut-être temps d'intégrer l'embryon humain dans le champ médical et scientifique avec tout le respect, toute la prudence, toute la rigueur, toutes les interrogations et tous les risques que cela implique. "L’ignorance permet la béatitude mais seule la connaissance peut nous libérer » [15]

\section{TIRÉS À PART}

\section{P. Jouannet.}

\section{RÉFÉRENCES}

8. Girardet A, Claustres M, Pellestor F. Typage génique des spermatozoïdes : application à l'êtude du génome. médecine/sciences 1996 ; 12: 1389-93.

9. Dickey RP, Taylor SN, Curole DN, Rye P. Male birth rates are influenced by the insemination of unselected spermatozoa and not by clomiphene citrate. Hum Reprod 1995 ; $10: 761-4$.

10. Liu P, Rose GA. Social aspects of 800 couples coming forward for gender selection of their children. Hum Reprod $1995 ; 10$ : $968-71$.

11. Tomkiewicz. Le fantasme d'enfant parfait ou l'heure du doute. In : Huber G, Jalbert $\mathrm{P}$, eds. Insémination artificielle : enjeux et problemes éthiques. Paris : John Libbey Eurotext, $1994: 159-66$.

12. Novaes S. Éthique et débat public. De la responsabilité médicale en matiere de procréation assistée. Raisons pratiques $1992 ; 3$ : 155-76. 13. Silber SJ, Ord T, Balmaceda J, Patrizio P, Asch RH. Congenital absence of the vas deferens. The fertilizing capacity of human epididymal sperm. N Engl J Med 1994; 323 : 1788-92.

14. Fagot-Largeault A. Procréation responsable. In : Sureau C, Shenfield F, eds. Aspects éthiques de la reproduction humaine. Paris : John Libbey Eurotext, 1995 : 3-17.

15. Petrinovich L. Human evolution, Reproduction and Morality. New York : Plenum Press 1995. 